\section{[gw22-e0279] THE ROLE OF RHOA/RHO KINASE SIGNALLING PATHWAY IN MYOCARDIAL FIBROSIS IN RATS WITH TYPE 2 DIABETES}

Hong Zhou, Yongjun Li The Second Hospital of Hebei Medical University, Hebei, China

\subsection{6/heartjnl-2011-300867.351}

Long-term diabetes can cause many serious complications. It is generally accepted that diabetes results in the development of diabetic cardiomyopathy independent of hypertension and coronary heart disease. Cardiac fibrosis is one of the major pathological processes of diabetic cardiomyopathy and is mainly manifested as proliferation of cardiac fibroblasts (CFs) and excess deposition of components of the extracellular matrix (ECM), such as collagens. Diabetes-induced cardiac remodeling may participate in diastolic and systolic dysfunction, leading to heart failure, and it is a key point for the inhibition of cardiac fibrosis to suppress increase in the proliferation of CFs and collagen synthesis. The previous studies about diabetic cardiomyopathy mainly focus on the changes of morphology and cytokines, the roles of signaling pathway in the pathogenesis of diabetic cardiomyopathy are few known. Rho is a small GTPase and is thought to be the molecular switch that mediates signals for various molecules. Rho kinase (ROCK) belongs to a family of serine/threonine protein kinases and is the first downstream effector of RhoA to be discovered. The study of RhoA/ROCK pathway is a foundation by which the molecular mechanisms are understood in cellular pathophysiological state. Several studies have clearly shown an important pathophysiological role for this pathway in human disease states, such as hypertension, stroke, chronic heart failure. It is reported that RhoA/ROCK pathway is associated with the chronic fibrosis of organs. c-jun $\mathrm{NH}_{2}$-terminal kinase (JNK) pathway is one of the major pathways of mitogen-activated protein kinases (MAPKs) and regulates various cellular functions, such as cell proliferation, differentiation and apoptosis. JNK is an important hinge of signal transduction from the cell surface to the nucleus and plays an important role in the fibrosis to which multifold cell growth factors lead. The previous studies have demonstrated 
that multifold growth factors participate in the development of myocardial fibrosis and that transforming growth factor $\beta 1$ (TGF $\beta 1$ ) is one of the most important pro-sclerotic growth factors. Smad protein is the TGF $\beta 1$ receptor-activated substrate in the endochylema. TGF $\beta 1$ activates its receptor and mediates signal transduction from the endochylema to the nucleus via phosphorylated Smad protein, which regulates gene and protein expression. TGF $\beta 1 / \mathrm{Smad}$ pathway has a major part in the fibrosis of organs. In the present study, we cultured CFs with high glucose (HG) in vitro and determined if the RhoA/ ROCK pathway is involved in HG-induced proliferation of CFs and collagen synthesis. Meanwhile, we also ascertained the relationship between the signal transduction of the RhoA/ ROCK and JNK pathway and the TGF $\beta /$ Smad pathway, with the objective of elucidating the molecular mechanism relating to diabetic cardiac fibrosis. The previous studies about diabetic cardiomyopathy mainly applied the animal model of type 1 diabetes rather than type 2 diabetes. However, more than $90 \%$ of patients with diabetes are afflicted with type 2 diabetes. Cardiovascular complications in patients with type 2 diabetes are major causes of diabetes related mortality. So, it is necessary to establish a animal model of type 2 diabetes, with the objective of studying diabetic cardiomyopathy. Several studies have reported that rats fed a high-fat diet (HFD)can develop insulin resistance. Low-dose streptozotocin (STZ) is known to induce a mild impairment of insulin secretion. A HFD with intraperitoneal injection of low-dose STZ for the induction of type 2 diabetes in rats has been found to be a good model for diabetes research. This type of model replicates the natural history and metabolic characteristics of type 2 diabetes in humans. We had successfully established type 2 diabetes in rats, and insulin resistance in diabetic rats was confirmed by the hyperinsulinemic-euglycemic clamp. Diabetic myocardial fibrosis may participate in diastolic and systolic dysfunction, leading to congestive heart failure, and it is closely related to a high prevalence of morbidity and mortality from cardiovascular events in diabetes. However, specific drugs for the prevention or treatment of diabetic cardiomyopathy are not available. Thus, a more thorough understanding of the pathogenesis of diabetic cardiomyopathy and the development of new pharmacologic treatments are needed. Fasudil is the only ROCK inhibitor practically available for human use and was approved for prevention and treatment of cerebral vasospasm following surgery for subarachnoid haemorrhage. Fasudil targets ROCK's ATP-dependent kinase domain and is equipotent in terms of inhibiting both ROCK1 and ROCK2. It is reported that fasudil leads to beneficial effects in patients with vasospastic angina and stroke. The previous studies about diabetic nephropathy have demonstrated that fasudil can prevent renal interstitial fibrosis. However, the roles of fasudil in diabetic myocardial fibrosis remain unknown. In the present study, we ascertained the effects of fasudil on diabetic myocardial fibrosis in vivo and the the molecular mechanism of action of fasudil. The objectives of the study were to determine whether activation of RhoA/ROCK is involved in myocardial fibrosis in diabetic cardiomyopathy in vivo and in vitro, and whether there is a relationship between RhoA/ ROCK and JNK pathway, and TGF $\beta /$ Smad pathway in diabetic hearts, with the objective of providing a novel target for the treatment of diabetic cardiomyopathy. Part 1: the role of RhoA/ROCK pathway in HG-induced proliferation and collagen synthesis in rat CFs.

Objectives To determine the role of RhoA/ROCK pathway in $\mathrm{HG}$-induced proliferation of CFs and collagen synthesis in vitro, and to investigate the molecular mechanisms of HG-induced proliferation of CFs and collagen synthesis.

Methods Rat CFs were cultured in DMEM (NG: 5.5 mM D-glucose; HG: $25 \mathrm{mM}$ D-glucose; OSM: $5.5 \mathrm{mM}$ $\mathrm{D}$-glucose+19.5 mM mannitol) with or without fasudil (50, $100 \mu \mathrm{M})$. Proliferation was measured by MTT assay. ELISA was used to measure the production of type-I collagen in media. ROCK1 and type-I procollagen mRNA expressions were assessed by RT-PCR. Intracellular type-I procollagen protein contents were evaluated by immunocytochemistry. The phosphorylation of MYPT1 was evaluated by Western blot analyses.

Results 1 Effects of HG and fasudil on the proliferation in CFs: As reflected by the optical density (OD) value in MTT assay, the proliferation of CFs was significantly promoted by HG (increased by $32.1 \%, \mathrm{p}<0.01$ vs NG group). In HG-stimulated cells, treatment with fasudil $(50-100 \mu \mathrm{M})$ significantly suppressed HG-induced increase in the proliferation of CFs in a dose-dependent manner (decreased by $12.4 \%(p<0.05)$ and $27.4 \%(p<0.01)$, respectively vs HG group). There was no significant difference in CF proliferation between OSM group and NG group $(p>0.05)$. These results suggested that HG promoted the proliferation in rat CFs and the effect of HG on the proliferation was independent of high osmotic pressure. Fasudil inhibited the proliferation of CFs in a dose-dependent manner. Treatment with fasudil $100 \mu \mathrm{M}$ was not cytotoxic to CFs. 2 Effects of HG and fasudil on the synthesis of type-I collagen: Compared with NG group, exposure of CFs to HG significantly upregulated mRNA expression of type-I procollagen $(p<0.01)$, which was inhibited by fasudil ( $p<0.01$ vs HG group). There was no significant difference in mRNA expression of type-I procollagen between OSM group and NG group ( $p>0.05$ ). We also observed the contents of intracellular type-I procollagen using immunocytochemical methods. Positive staining for type-I procollagen, which was presented as filemot granulations, was expressed in the fibroblastic endochylema. Very strong positive staining for type-I procollagen was found in the cells of HG group. The contents of intracellular type-I procollagen in HG group were markedly higher than those in NG group $(p<0.01)$. Fasudil $(50,100 \mu \mathrm{M})$ inhibited HG-induced increase in contents of type-I procollagen in a dose-dependent manner $(p<0.05, p<0.01$ vs HG group). There was no significant difference in the contents of type-I procollagen between OSM group and NG group ( $p>0.05$ ). To assess the effects of $\mathrm{HG}$ and fasudil on the extracellular matrix production in CFs, type-I collagen accumulation in media was determined by ELISA. Exposure of CFs to HG significantly induced a 2.5-fold increase in accumulation of type-I collagen ( $p<0.01$ vs NG group). Treatment CFs with fasudil (50-100 $\mu \mathrm{M})$ significantly suppressed the increase in accumulation of type-I collagen induced by HG in a dose-dependent manner $(p<0.05, p<0.01$ vs HG group); $100 \mu \mathrm{M}$ fasudil reduced accumulation of type-I collagen by $\sim 61.6 \%$ ( $p<0.01$ vs HG group). There was no significant difference in accumulation of type-I collagen between OSM group and NG group ( $p>0.05)$. These results suggested that HG induced increase of type I collagen synthesis, which was inhibited by fasudil. The change of osmolarity did not affect type I collagen synthesis. 3 Effects of HG and fasudil on RhoA/ROCK pathway: Compared with NG group, exposure of CFs to HG induced a significant upregulation of ROCK1 mRNA expression $(p<0.01)$, which was significantly repressed by high-dose fasudil $(100 \mu \mathrm{M})(\mathrm{p}<0.01 \mathrm{vs} \mathrm{HG}$ group) but not by low-dose fasudil $(50 \mu \mathrm{M})(\mathrm{p}>0.05)$. ROCK can inhibit myosin phosphatase by phosphorylating myosin phosphatase target 
subunit 1 (MYPT1). We therefore measured the phosphorylation of MYPT1 as a maker of ROCK activity. Exposure of CFs to HG significantly increased the phosphorylation of MYPT1 ( $p<0.01$ vs NG group). Fasudil inhibited HG-induced increase in phosphorylation of MYPT1 ( $p<0.01$ vs HG group). There was no significant difference in the mRNA expression of ROCK1 and phosphorylation of MYPT1 between OSM group and NG group $(p>0.05)$. These results suggested that HG activated RhoA/ROCK pathway, and the change of osmolarity was not responsible for ROCK activation.

Conclusions These data suggest that HG promotes the proliferation and collagen synthesis in rat CFs. ROCK activation is essential for the proliferation of CFs and collagen synthesis induced by HG. Part 2: the effects of RhoA/ROCK pathway on JNK and TGF $1 /$ Smad pathways in HG-induced proliferation and collagen synthesis in rat CFs.

Objectives To investigate whether RhoA/ROCK pathway regulates JNK and TGF $\beta /$ Smad pathways in HG-induced proliferation of CFs and collagen synthesis in vitro, and to elucidate the molecular mechanism of action of fasudil on the functions of CFs induced by HG.

Methods Rat CFs were cultured in DMEM (NG: 5.5 $\mathrm{mM}$ D-glucose; HG: $25 \mathrm{mM}$ D-glucose; OSM: $5.5 \mathrm{mM}$ D-glucose $+19.5 \mathrm{mM}$ mannitol) with or without fasudil (50, $100 \mu \mathrm{M})$. JNK mRNA expressions were assessed by RT-PCR. The phosphorylation of JNK and $\mathrm{Smad} 2 / 3$, as well as c-jun and TGF $\beta 1$ protein levels, were evaluated by Western blot analyses.

Results 1 Effects of HG and fasudil on the JNK pathway: To further investigate the signal crosstalk between the RhoA/ ROCK pathway and JNK pathway, we observed the effects of HG and fasudil on the JNK signalling pathway. As the substrate of JNK, c-jun has been identified as a mediator of cell proliferation in response to JNK activation. Compared with NG group, exposure of CFs to HG significantly upregulated JNK mRNA expression and increased the phosphorylation of JNK and $c$-jun protein expression $(p<0.01)$, which were markedly blocked by fasudil ( $<<0.01$ vs HG group). Fasudil had no effects on JNK pathway in CFs under NG conditions. There were no significant differences in JNK mRNA expression and phosphorylation of JNK between OSM group and NG group ( $p>0.05$ ). These results suggested that HG activated JNK pathway, which was inhibited by fasudil. High osmotic pressure was not responsible for JNK activation. 2 Effects of HG and fasudil on the TGF $\beta / S m a d$ pathway: In addition to the JNK pathway, collagen gene expression is regulated by the Smad signalling pathway, which is activated by transforming growth factor- $\beta 1$ (TGF $\beta 1$ ). In addition to examining TGF $\beta 1$ protein expression, We assessed the biological effects of TGF $\beta 1$ by examining one of its specific intracellular actions: the phosphorylation of the TGF $\beta$ receptor-activated protein, Smad2/3. Compared with NG group, exposure of CFs to HG led to a significant increase in TGF $\beta 1$ protein expression and the phosphorylation of $\operatorname{Smad} 2 / 3(p<0.01)$, which was attenuated by fasudil ( $p<0.01$ vs HG group). Fasudil had no effect on TGF $\beta 1$ protein expression and the phosphorylation of Smad2/3 in CFs under NG conditions. There was no significant difference in TGF $\beta 1$ protein expression and the phosphorylation of Smad2/3 between OSM group and NG group ( $p>0.05)$. These results suggested that HG activated TGF $\beta 1 / \mathrm{Smad} 2 / 3$ pathway, which was inhibited by fasudil. High osmotic pressure was not responsible for TGF $\beta 1 / \mathrm{Smad} 2 / 3$ activation.

Conclusions The present study suggests ROCK activation is specifically required for HG-induced JNK and TGF $\beta 1 / \mathrm{Smad} 2 / 3$ activation in rat CFs. JNK and Smad2/3 are the downstream signalling molecules of ROCK. Fasudil was capable of suppressing HG-induced CFs proliferation and collagen sythesis, which might be associated with in part the inhibition of JNK and TGF $31 /$ Smad pathways. Part 3: the role of RhoA/ROCK pathway in myocardial fibrosis in a rat model of type 2 diabetes and protective effects of fasudil on diabetic heart.

Objectives To understand the roles of RhoA/ROCK, JNK and TGF $\beta /$ Smad pathways in myocardial fibrosis in rats with type 2 diabetes, and to ascertain the effects of fasudil on myocardial fibrosis in vivo.

Methods A model of type 2 diabetes in rats was established by a HFD combined with low-dose STZ (30 mg/kg intraperitoneally). At week 10, insulin resistance in rats fed a HFD combined with low-dose STZ was shown by the hyperinsulinemic-euglycemic clamp. Fasting insulin (FINS), fasting blood glucose (FBG), total cholesterol (TC) and triglyceride (TG) were measured. Diabetic rats were randomly divided into untreated diabetic rats and treated diabetic rats that received fasudil (10 mg/kg/day intraperitoneally) for 14 weeks. Systolic arterial blood pressure (SABP) was measured monthly by tailcuff micro-photoelectric plethysmography. At week 24, in vivo cardiac function was measured, including heart rate (HR), left ventricular systolic pressure (LVSP), left ventricular enddiastolic pressure (LVEDP) and maximum change velocity of left ventricular pressure in the isovolumic contraction or relaxation period ( \pm dP/dtmax). FINS, FBG, TC, TG and glycosylated haemoglobin (HbA1c) were measured. The morphology of the myocardium was observed by light microscope and transmission electron microscope (TEM). Masson staining for collagen and immunohistochemistry staining for type-I collagen and type-U collagen were performed. The mRNA expression of ROCK1, JNK, TGFß1, type-I and type-Ш procollagen were assessed by RT-PCR. The phosphorylation of MYPT1, JNK and Smad2/3, as well as the protein levels of TGF $\beta 1$ and c-jun, were evaluated by Western blot analyses.

Results (1) Changes of body weight (BW): At week 10, the BW of diabetic rats was significant higher than that of control rats (328.2 $\pm 21.72 \mathrm{~g}$ vs $276.65 \pm 33.18 \mathrm{~g}, \mathrm{p}<0.01)$. At week 24 , the BW of untreated diabetic rats and fasudil-treated diabetic rats were significant lower than that of control rats $(297.06 \pm 35.11 \mathrm{~g}$ and $301.77 \pm 37.39 \mathrm{~g}$ vs $349.10 \pm 40.01 \mathrm{~g}$, respectively, $\mathrm{p}<0.01)$. There were no significant differences in BW between untreated diabetic rats and fasudil-treated diabetic rats $(297.06 \pm 35.11 \mathrm{~g}$ vs $301.77 \pm 37.39 \mathrm{~g}, \mathrm{p}>0.05$ ). (2) Blood biochemical parameters: At week 10, compared with control rats, diabetic rats had higher values of FBG, FINS and TG $(p<0.01)$, but there was no significant difference with respect to TC levels ( $p>0.05)$. At week 24 , compared with control rats, the levels of FBG, FINS, HbA1c, TC and TG were significantly increased in untreated diabetic rats and fasudil-treated diabetic rats $(p<0.01)$. There were no significant differences in these indices between untreated diabetic rats and fasudil-treated diabetic rats ( $p>0.05)$. There were no significant difference in SABP among the three groups ( $p>0.05$ ). (3) Insulin sensitivity: At week 10, the glucose infusion rate (GIR) of diabetic rats was lower than that of control rats $(5.89 \pm 1.05 \mathrm{mg} / \mathrm{kg} \mathrm{min}$ vs $11.40 \pm 1.24 \mathrm{mg} / \mathrm{kg} \mathrm{min}, \mathrm{p}<0.01)$. The results suggested decreased insulin sensitivity and the development of insulin resistance in diabetic rats. At week 24, the homeostasis model assessment for insulin resistance (HOMA-IR) was calculated as FBG $\times$ FINS/22.5. Compared with control rats, HOMA-IR were significantly increased in untreated diabetic rats and fasudil-treated diabetic rats $(92.72 \pm 17.42$ and $81.43 \pm 12.81$ vs $13.70 \pm 3.91$, respectively, 
$\mathrm{p}<0.01)$. There were no significant difference in HOMA-IR between untreated diabetic rats and fasudil-treated diabetic rats $(92.72 \pm 17.42$ vs $81.43 \pm 12.81, \mathrm{p}>0.05)$. (4) Parameters of cardiac function: At week 24, compared with control rats, untreated diabetic hearts showed significantly reduced LVSP and $\pm \mathrm{dP} /$ dtmax $(101 \pm 17 \mathrm{~mm} \mathrm{Hg}$ vs $139 \pm 21 \mathrm{~mm} \mathrm{Hg}, \mathrm{p}<0.05 ; 3746 \pm 402$ $\mathrm{mm} \mathrm{Hg} / \mathrm{s}$ vs $6909 \pm 597 \mathrm{~mm} \mathrm{Hg} / \mathrm{s}, \mathrm{p}<0.01 ; 3417 \pm 409 \mathrm{~mm} \mathrm{Hg} / \mathrm{s}$ vs $6811 \pm 625 \mathrm{~mm} \mathrm{Hg} / \mathrm{s}, \mathrm{p}<0.01)$ whereas LVEDP was significantly increased $(10.99 \pm 2.21 \mathrm{~mm} \mathrm{Hg}$ vs $3.51 \pm 0.72 \mathrm{~mm} \mathrm{Hg}$, $\mathrm{p}<0.01)$. Compared with untreated diabetic hearts, treatment with fasudil significantly enhanced LVSP, $\pm \mathrm{dP} / \mathrm{dtmax}(131 \pm 24$ $\mathrm{mm} \mathrm{Hg}$ vs $101 \pm 17 \mathrm{~mm} \mathrm{Hg}, \mathrm{p}<0.05 ; 6517 \pm 601 \mathrm{~mm} \mathrm{Hg} / \mathrm{s}$ vs $3746 \pm 402 \mathrm{~mm} \mathrm{Hg} / \mathrm{s}, \mathrm{p}<0.01 ; 5937 \pm 513 \mathrm{~mm} \mathrm{Hg} / \mathrm{s}$ vs $3417 \pm 409$ $\mathrm{mm} \mathrm{Hg} / \mathrm{s}, \mathrm{p}<0.01)$ and decreased LVEDP $(4.88 \pm 1.17 \mathrm{~mm} \mathrm{Hg}$ vs $10.99 \pm 2.21 \mathrm{~mm} \mathrm{Hg}, \mathrm{p}<0.01)$. There were no significant difference in HR among the three groups ( $p>0.05)$. (5) Heart weight (HW) and the ratio of $\mathrm{HW}$ to BW (HW/BW): Compared with control rats, $\mathrm{HW}$ and $\mathrm{HW} / \mathrm{BW}$ were significantly increased in untreated diabetic rats $(p<0.01)$. Compared with untreated diabetic rats, treatment with fasudil significantly decreased $\mathrm{HW}$ and $\mathrm{HW} / \mathrm{BW}(\mathrm{p}<0.01)$. There were no significant differences in HW and HW/BW between fasudil-treated diabetic rats and control rats $(p>0.05)$. (6) The contents of collagen in hearts: The contents of collagen in untreated diabetic hearts were higher than those in the control hearts $(3.664 \pm 0.243 \mu \mathrm{g} /$ $\mathrm{mg}$ vs $2.216 \pm 0.122 \mu \mathrm{g} / \mathrm{mg}, \mathrm{p}<0.01)$. Compared with untreated diabetic hearts, treatment with fasudil significantly reduced the contents of collagen in the hearts $(2.292 \pm 0.103 \mu \mathrm{g} / \mathrm{mg}$ vs $3.664 \pm 0.243 \mu \mathrm{g} / \mathrm{mg}, \mathrm{p}<0.01)$. There were no significant difference in the contents of collagen in the myocardium between fasudil-treated diabetic rats and control rats $(2.292 \pm 0.103 \mu \mathrm{g} /$ mg vs $2.216 \pm 0.122 \mu \mathrm{g} / \mathrm{mg}, \mathrm{p}>0.05)$. (7) HE staining: In the control rats, cardiac myocytes lined up in order and their structure was normal. There were a little fibroblasts whose nucleus were fusiform. Diabetic rat hearts showed disarray of myocytes, disorganisation, cardiac myocyte hypertrophy, increased fibroblasts in the interstitium and perivascular space. The cardiac morphology of fasudil-treated diabetic rats was similar to that of control rats. (8) The results of TEM observations: The ultrastructure of the myocardium in control rats shows a normal array of sarcomere, regular myofibrillar organisation with evident $Z$ lines. The myocardium in untreated diabetic rats showed dense packages of collagen fibrils between cardiomyocytes, mitochondrial swelling, and large areas of cytoplasm had lost their regular myofibrillar organisation. The cardiac morphology of fasudil-treated diabetic rats was similar to that of control rats, regularly organised cytoplasm with numerous myofibrils arranged in parallel. (9) Masson staining: The deposition of collagen in the myocardial interstitium from untreated diabetic hearts was significantly increased compared with that seen in control rats (area: $10.15 \pm 0.79 \%$ vs $2.42 \pm 0.33 \%, \mathrm{p}<0.01$ ). However, treatment with fasudil significantly reduced the deposition of collagen in the myocardial interstitium compared with that seen in untreated diabetic rats (area: $2.20 \pm 0.31 \%$ vs $10.15 \pm 0.79 \%, p<0.01$ ). (10) Immunohistochemistry staining: An increase in deposition of type-I and type-III collagen was shown in untreated diabetic rats compared with that seen in control rats (area: $38.4 \pm 8.2 \%$ and $15.9 \pm 4.6 \%$ vs $11.2 \pm 2.9 \%$ and $4.3 \pm 1.5 \%$, respectively, $\mathrm{p}<0.01$ ). This increase was completely inhibited by treatment with fasudil (area: $13.7 \pm 4.1 \%$ and $5.1 \pm 2.3 \%$ vs $38.4 \pm 8.2 \%$ and $15.9 \pm 4.6 \%$, respectively, $\mathrm{p}<0.01)$. (11) Gene expression in LV tissues: Compared with control rats, mRNA expression of type-I, type-III procollagen, JNK and TGF $\beta 1$ were significantly upregulated in hearts from untreated diabetic rats $(\mathrm{p}<0.01)$, this upregulation was inhibited by treatment with fasudil $(p<0.01$ vs untreated diabetic rats). Compared with control rats, mRNA expression of ROCK1 was significantly increased in the hearts of untreated diabetic rats $(p<0.01)$. But there was no significant difference in ROCK1 mRNA expression between untreated diabetic rats and fasudil-treated diabetic rats ( $p>0.05)$. (12) Protein expression in LV tissues: Compared with control rats, the phosphorylation of MYPT1, JNK and Smad2/3, as well as the protein levels of TGF $\beta 1$ and c-jun, were significantly enhanced in hearts from untreated diabetic rats $(p<0.01)$, which were attenuated by treatment with fasudil ( $p<0.01$ vs untreated diabetic rats).

Conclusions The present study suggests that a critical role of the RhoA/ROCK cascade in remodeling of the myocardial matrix in diabetic rats. Fasudil suppresses myocardial fibrosis in diabetic rats at least in part via the inhibition of JNK and TGF $\beta /$ Smad pathways, and these beneficial structural effects of fasudil are independent of blood pressure and glycemic control. Inhibition of the Rho/ROCK pathway may be a novel therapeutic target for prevention of diabetic cardiomyopathy. 